\title{
A SURVEY- HETEROGENITY AND MOBILITY BASED ROUTING PROTOCOL IN WSN
}

\author{
Ravi Yadav ${ }^{1}$, Awadhesh Kumar², Rupendra Kumar ${ }^{3}$ \\ ${ }^{1}$ Research Scholar, Kamla Nehru Institute of Technology, India, ravics85@gmail.com \\ ${ }^{2}$ Associate Professor, Kamla Nehru Institute of Technology, India, awadheshkumar.knit@gmail.com \\ ${ }^{3}$ Research Scholar, Kamla Nehru Institute of Technology, India, rupendra5989@gmail.com
}

\begin{abstract}
The latest advancements in wireless communication are more emphasis on delivering sensitive info to destination node under several constraints such as latency, energy, reliability, stability, and security. So there is rise of Wireless Sensor Network Technology[1] .Though it is possible to assimilate sensing and computing units along with transceiver(transmitter \& receiver) and power supply to a single entity called as Sensor Node (SN). The combination of SNs constitute a type of network known as Wireless Sensor Networks (WSNs)[1]. Several researched proposals on WSNs have mainly emphasis on energy minimization of the Base Station (BS) for communication process. Although clustering is the most well-known technique for the enrichment of network lifetime by effective energy utilization of sensor node, but heterogeneity issue of sensor node is another interesting aspect which can be considered to save the energy consumption of sensor nodes in the network field. Mobility Issue is also one of the major aspect in determining performance of the network. In this paper considering all these issues, , we have tried our best to categorized different heterogeneous routing protocols for WSNs based upon some predefined parameters .Different performance evaluation metrics such as- network lifetime, Number of Heterogeneity Level, Cluster Head Selection(CHSelection), Energy Efficiency, Stability and Mobility. The extensive categorization gives insights to various readers to select one of the protocols from various categories based upon its advantage over the others.
\end{abstract}

Keywords: - Heterogeneity, Cluster Head Selection, Mobility, Heterogeneous Routing Protocol, Threshold Value.

\section{INTRODUCTION}

Wireless Sensor Network is now emerged as one of the most vital technologies in twentieth century .In past decade it has gain tremendous attention from both educational and industrial field all over the world. A Wireless Sensor network consists of large number of sensor node that are deployed in area of interest in random fashion. Sensor node is small sized autonomous device that consists of four units, i.e sensing, processing, communication and power supply .Sensor node are low-power, low-cost, and multifunctional in nature. A sensor node is embedded with microprocessors, radio receiver, and power unit for sensing ,computation, and communication process. All this units are embedded and integrated on a single or multiple board chip .Sensor node communicate in a range of distance via wireless medium and collaborate to accomplish the common tasks like tracking, monitoring, observing and process controlling .Sensor node have sensors which is used to monitor both physically and environmental situations such as movement, temperature, heat, pressure, vibration etc. According to various application of network sensors may range from hundreds to thousands in numbers deployed in random fashion. After the deployment of sensor node in an area, the sensor node must autonomously organize themselves for communication process to continue in network via wireless mode. Here sensor node act both as router as well as normal sensing node. All sensing node must sense the information from environment and transmit to base station(BS) also known as sink node. Base station collect the information from all sensor node, analyze it and perform some computation which yields information that are human understandable. Since base station have unlimited source of energy power so it should implement various algorithm and protocols which result in enhancement in lifetime of sensor node and network lifetime as well energy efficient. WSNs consists of dense level of sensor node deployment, unreliability of sensor node and severe constraints of power, memory lead to development of various application of WSNs such as military application, environmental observation, medical application, disaster management, habitat tracking, home automation etc[2]. 


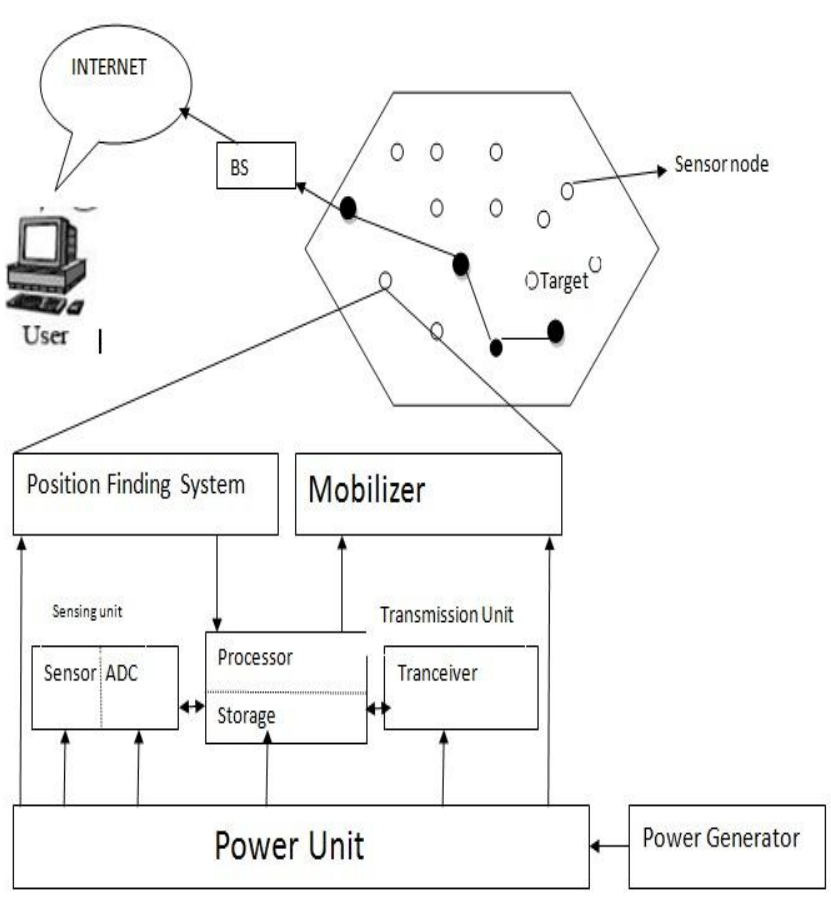

Fig 1. An Architecture of WSNs.

Generally ,WSNs consists of large number of sensor node deployed in random fashion so routing issue is one of major issue in WSNs. Routing are more complex in WSNs as node are power limited, self organized, no addressing schemes etc. all this makes routing more complex. On solution to Routing is Clustering. In Clustering we divide our whole network into some small-small cluster and select one cluster head for each cluster .Each cluster head aggregates information from all other nodes and transmit it to the sink node .So by this approach only few nodes is allow to send data continuously so it saves battery power of many sensor nodes. But Selection of cluster head is major issue in clustering .If we assume homogenous network (all node compose same energy level), any node can be chosen as cluster head . But in case of different energy level of sensor node (heterogeneous network) selection of cluster head is major issue. In Heterogeneous routing protocol we choose cluster head periodically to increase the overall lifetime of network. In Heterogeneous network we have different level of heterogeneity like two level heterogeneity, three level heterogeneity and multi-level heterogeneity.

Mobility brings new challenges in routing protocols in WSNs. So routing mechanism should be energy efficient for guaranteed info delivery between sensor node and mobile sink node .Since we mostly considered static sink node which leads to various problem called energy-sink holes problem where nodes near static sink nodes get degrades very rapidly. We also consider this aspect for future aspect.

In this paper, section 2 gives an overview of designing challenge and routing issues of WSNs. Section 3 gives an overview of heterogeneous routing protocols .Section 4 give an idea of mobility based routing protocols and last section contain the conclusion of this survey paper and future scope of the WSNs.

\section{NETWORK DESIGN CHALLENGE AND ROUTING ISSUES IN WSNS}

WSNs faces severe network constraints due to unreliability of sensor node ,dynamic topology. The several network resource constraints are limited energy, bandwidth ,memory ,computation capability etc. Following are some design challenges which are to be considered while designing routing protocols in WSNs.

Limited Functional Capability :-A sensor node has small memory ,limited battery power, and limited processors for performing sensing, computation and communication process. This limitation also constraints the functional capabilities of sensor nodes. So a good algorithm must be used for efficient utilization of network resources by sensor node.

Limited Energy Source:- A sensor node has limited battery power for carrying out its functions .So an power efficient algorithm must be required for increasing lifetime of network. Sensor node must be in sleeping mode when its doesnot participate in transmitting process.

Area of deployment:- Sensor node deployed in any area must be in such a way that its location can be traced and monitor by base station and deployment must be within range of base station. We use Global Positioning System(GPS) to track down the location of sensor node.

Limited Resources Source:-the network resources such as memory ,bandwidth, power supply are limited which leads to more challenging issues while designing any routing protocols for WSNs.

Dense and Random mode of node deployment:- In WSNs the sensor nodes are deployed in huge number ranging from hundreds to thousands in an deployed area. The sensor node are densely deployed in random fashion in an environmental area. If the distribution of sensor nodes are not uniformly, then optimal clustering is used for energy effecting routing.

Heterogeneity Environment:- Since network may be homogenous or heterogeneous. When we consider heterogeneous network all sensor nodes posses different energy level. So we suggest Clustering technique in Environment Issue .But Selection of Cluster Head is also one big factor while designing routing protocols.

Mobility Issues:-Since Sensor nodes are mostly mobile in nature but what happen when sink node is also mobile in nature. Due to static sink node we have problem like energysink holes problems. We also keep these issue for future aspects.

Unreliable Environment and unpredictable network characteristics :-A WSNs frequently used in dynamic and unreliable environment like military base, forests, no possible wired connection area etc. Due to frequent changes 
in topology due to node deletion , addition ,degradation and node failures its very challenging to decide which routing protocol we used. Also we are using wireless medium which are prone to noise, error, latency and interference. So all this factor makes designing issue more challenging.

Data aggregation:- As sensor node nodes sense and gathers redundant data from various other sensor node, its duty is to aggregates all data and transform only relevant data to base station for both energy efficient and optimization of data transferred. So Data aggregation is an significant factor for designing issue .

Scalability:-Since while setting up any WSNs we are not sure that how many sensor node we are required.So that the network scalability is one major issue which should be maintained so that in future if we want to increase number of sensor node without affecting network performance it can be easily done.

Latency:-Latency refers to the delay in time when a packet sent from sender side until its acknowledged at receiver side .low latency is significant for network throughput

Network lifetime:-Network life refers to the time between start of first node until degrades of all nodes in WSNs. So the routing protocols must be energy efficient for long network lifetime

Redundancy:-Due to unreliable network WSNs must have high redundant sensor nodes so that failure of any node doesn't affect overall network performance

Cost Effective:-the routing mechanism should be cost effective so sensor node production cost must be as low as possible

Fault tolerant:-Due to frequent failure and degradation of nodes fault of network in most occurring problem .So routing mechanism should be fault tolerant.

\section{HETEROGENEOUS ROUTING PROTOCOLS-}

WSNs are characterized by its unreliable topology and unpredictable network characteristics which make more challenging issues while designing routing protocols for WSNs. Routing protocols are set of rules and standards which are to be followed by two or more station (nodes) for communication process. Routing is the most challenging issue in WSNs due to inadequate battery power supply ,restricted bandwidth, limited resources and wireless medium which are more intent to error and noise attack. So routing protocols which is preferable for WSNs must be energy efficient and data guaranteed delivery to destination node. Earlier we used direct communication mechanism for routing protocols in WSNs .In this mechanism, all the sensor nodes sense the data and continuously transmits directly to the sink node .this is simplest approach. The drawback of this approach is that the sensor nodes which are to be found far away from the sink node gets degrades first and dies first, thereby decreasing the network lifetime of the network. Second approach we used here is multi hopping approach. In this approach instead of each sensor nodes directly transmit it data to base station, the sensor node transmit it data to next neighbor sensor nodes which is more close to sink node .then this sensor node transmit it next neighbor node which is close to sink node .This process continue until it is single hop distance from sink node. The drawback of this approach is that the sensor nodes which are close to sink node get degraded first as it is always in active mode by continuously receiving data from other station and transmitting it to the base station.

Energy Efficient is the most significant criteria that must be fulfill while designing any routing protocols for WSNs. Clustering is one of the mechanism by which we can design energy efficient routing mechanisms[3]. In this approach whole network is divided into small-small cluster , and in each cluster we select one node as cluster head. The main objective of cluster head selection is instead of transmitting data by all sensor nodes to base station only cluster head is allow to transmit it sink node. Cluster head node gather all the data from other sensor nodes in cluster, aggregates it and transmit to the sink node. Thus, only few nodes are required to transmit the data over a long distance while rest of them are required to transmit in a short range of distance result in saving more energy and enhancing the overall network lifetime period. Clustering is suitable for both homogenous and heterogeneous WSNs. Homogenous network are type of network in which all the sensor nodes have same level of energy(battery power).LEACH is common protocol for homogenous network. Though all the nodes posses same energy level selection of cluster head is easy and random in sense. Heterogeneous network are network in which different nodes contains different energy level .Energy level may be two-level, three-level, and may be multi-level in nature depend on the area of deployment .All the heterogeneous protocol are designed based on clustering mechanism, in which cluster head are select periodically on different criteria. Since after some period of working homogenous network behaves as heterogeneous network. So choosing of cluster head is one major factor while designing heterogeneous routing protocols.

The First work presented focusing on the behavior of clustering in terms of heterogeneity parameter in clustered WSNs was given by W.R Heinzelman[4]. In His work, he tried to elect cluster head on the basis of how much energy level left in each sensor.After analyzing this attempt of method, the drawback pop out suggesting that choosing of cluster head is made per round and he assumed that overall energy left in network is known. Assumption of global knowledge of how much amount of energy left in the network at any time makes this attempt of method difficult to implement. Even A centralized version of this method would very complex and slow as acknowledgement should be reliably delivered to each sensor nodes after every round of transmission.

Later Duarte-Melo and Liu presented their work which focused on the performance and energy consumption of WSNs[5]. In their work they take two category of sensor 
nodes i.e normal node and overlay node .Normal nodes are simply sensor nodes which sense their area of environment and report to overlay node and are less powerful than overlay node(Energy term). While Overlay nodes are fewer in number but are more powerful than normal nodes. All other node (normal nodes) have to report to overlay nodes and these overlay nodes aggregates the data received by other node and transmit to sink node. The drawback of this work is that there is static election of cluster heads among the types of sensor nodes, which result in sensor nodes which are at long distance from overlay node dies first result in degrading performances of WSNs. Estimation of optimal number of overlay nodes are very difficult to predict along with heterogeneity parameter.

Later in [6] Mhatre and Rosenberg present a comparative study on both homogenous and heterogeneous WSNs based on cost factor .In their study, optimal distribution among nodes is estimated, but again heterogeneity make it difficult to implement as it is result of network operation. They also examined and analyzed the case of multi-hopping routing within each cluster(known as M-LEACH).Again disadvantage of this method there is that only powerful nodes have probability of becoming cluster head(although all powerful nodes are not used in each round).also there are many assumption which is to be consider while implementing and applicable in largely populated network. Later we use GPS [7] system for power aware routing protocols increasing cost of network and valid for only large and high cost network.

Since all heterogeneous routing protocols uses cluster head algorithm so this algorithm is broken into step called round .A round means the time at which all alive nodes are ready to transmit their data to sink node. In each round, sensor nodes decide which sensor nodes is to be elected as sensor node based on threshold suggested for cluster heads of the network(pre determined) and the number of times that node is selected as cluster head. For making this decision initially sensor node is allowed to choose a random between 0 and 1.If the number selected is less than a Threshold Value T(s), then that sensor node become cluster head for that current round. the threshold value is set as:

$$
\begin{gathered}
\mathrm{T}(\mathrm{S})=\frac{p}{1-P\left(\bmod \frac{1}{D}\right)} \text { if } \mathrm{Si} € \mathrm{G} \\
0, \quad \text { otherwise }
\end{gathered}
$$

Where $\mathrm{p}=$ probability of node $\mathrm{si}$ and this value change for every protocols

$\mathrm{r}=$ current round

$\mathrm{G}=$ set of nodes which doesn't elected se cluster head in last $1 /$ popt round

Using this threshold value each node become cluster head at some point within 1/popt round.

Here are some performance metrics listed below which are used for evaluation of various routing protocols.

Stability period:-It can be defined as time interval between (in terms of number of rounds) the start of network operation untill first node of network dies.

Network lifetime:- It can be defined as time between the start of network operation until all the nodes in network dies. It is also calculated in term of number of rounds.
Throughput:- Throughput can be defined as total amount of data received by sink node during whole network life time.

\section{Types of routing protocol.}

Following are list of heterogeneous routing protocols :-

- SEP

- DEEC

- DDEEC

- TSEP

- ETSSEP

- EDEEC

- EDDEEC

- BEENISH

\subsection{Sep (Selection Election Protocol)}

Georgios Smaagdakis, Ibrahim Matta and Azer Bestavros [8] proposed SEP which contain two type of nodes known as normal nodes and advance nodes respectively. The advanced node have energy $(1+a)$ times more energy than normal nodes. Here we consider two -level heterogeneity where every sensor nodes independently elect himself as cluster head based on initial energy level in reference to other nodes.

Probability of node to be elected as cluster head is given by equation

$\mathrm{P}=\frac{\text { Popt }}{P+a m} \quad$ for normal node

$\frac{\operatorname{Popt}(1+a)}{(1+a m)}$ for advance node

Popt=optimal probability of node to be elected as cluster head.

SEP protocols are dynamic in nature and doesnot require any global-knowledge for election of cluster head after every round. It is also scalable in nature as it doesn't require any exact location of each node in deployed area.

\subsection{DEEC(Distributed Energy-Efficient Clustering)}

DEEC protocol was propsed by Li Qing,Qingxin Zhu and Mingwen Wang [9] which is based on two-level heterogeneity i.e, two levels of energy-normal nodes and advance nodes same as in SEP protocol and it have better stability period than SEP. In this protocol, the Election of cluster head is based on probability i.e, based on ratio of residual energy of each sensor node and average energy of the network. The period of node being a cluster head are different for each node as it is based on initial energy and residual energy. The nodes with having high initial energy and residual have more probability to become cluster head . This give an idea that the advance node have high probability of electing as cluster head as compared to normal nodes. The probabilities of electing as cluster head for normal nodes and advance nodes are given by eqn. $\mathrm{P}=\frac{P \text { opt Ei }(\mathrm{r})}{(p+\operatorname{an}) E(\eta)}$ for normal node 
$\operatorname{Popt}(1+a) \operatorname{Ei}(\mathrm{r})$

$(1+a m) E(r) \quad$ for advance node

$\mathrm{Ei}(\mathrm{r})=$ residual energy of node $\mathrm{Si}$ at round $\mathrm{r}$

$\dot{\bar{E}}(\mathrm{r})=$ average energy at round of network

$\mathrm{r}=$ current round of network.

Simulation results shows that DEEC yields longer lifetime and efficient than earlier protocols.

\subsection{Ddeec (Developed Distributed Energy-Efficient}

\section{Clustering)}

Brahim Elbhiri,Saadane Rachid, Sanaa El fkihi and Driss Aboutajdine [10]proposed this protocol which was also based on two level heterogeneity and yields $30 \%$ and $15 \%$ better than SEP and DEEC respectively in context of network lifetime and Stability period. This protocol also remove the drawbacks of DEEC protocols. DEEC follows the rotating epoch of each node which is different for every node. As the round number of rotating epochs depend on the initial energy and residual energy of each sensor node .So the advance node have high probabilities for electing as cluster head as it have high initial energy and residual energy. This electing procedure of DEEC result in improving network life time and stability period but also result in penalizing effect. In penalizing effect the advance node residual energy degraded rapidly and soon they are like as normal node. Thus DDEEC remove this effect by balancing the election of cluster head between advance node and normal node. Initially advance node is selected as cluster head ,as energy of advance node get degraded and it have energy level in range of normal nodes, it uses same election probability function of normal node. The election probabilities are given by eq

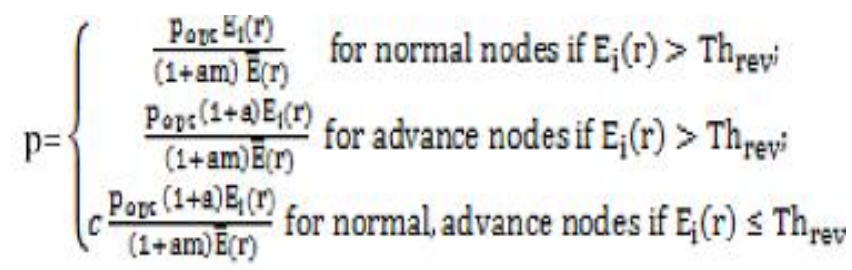

Where Threv $=b E 0$, where $b €(0,1)$ ad E0 is initial energy of network. When $b=0$ it behaves as traditional DEEC Since all advance node cannot be cluster head same thing is also similar in normal only few get chances to be elected as cluster head. Since value of $b$ is not fixed we find nearest of $\mathrm{b}$ value which yields best result. Value of $\mathrm{c}$ controls the number of cluster heads. If $\mathrm{c}$ value is high or zero it behaves like direct communication. So we have to choose optimal value of $\mathrm{c}$ for better simulation results.

DDEEC protocol yields better performance than DEEC protocol by eliminating penalizing effect.

\subsection{Tsep (Threshold Sensitive Stable Election}

\section{Routing Protocol)Protocol:-}

This protocol was proposed by A.Kasaf, N. Javid, Z.A Khan, I.A Khan[11] based on three level of heterogeneity i.e normal node, intermediate node and advance node , where energy level of advance node is highest while least is of normal node. This protocol comes under the category of reactive routing protocols means transmission of data takes place only when there is an occurrence of any event when specified threshold is achieved. IN this type of protocol, transmission energy is more consumes than sensing energy. This protocol assume that there are total n number of nodes, $\mathrm{m}$ is proportion of advanced node where $\mathrm{b}$ is proportion of intermediate node. Energy of advanced node is $(1+\alpha)$ times that of normal node while energy of intermediate node is $(1+\mu)$ times that of normal nodes and relation between $\mu=\alpha / 2$. The probabilities for cluster head of nodes are as follow:-

$$
\begin{gathered}
\text { Pnrm }=\frac{\text { Popt }}{(1+\alpha \cdot m)+b \mu} \\
\text { Pint }=\frac{\text { popt } \cdot(1+\mu)}{(1+m \cdot \alpha+b \mu)}
\end{gathered}
$$$$
\operatorname{Padv}=\frac{\text { popt } \cdot(1+\alpha)}{(1+m \cdot \alpha+b \mu)}
$$

For these nodes we have different calculation formulas of threshold value based on their probability.

$$
\begin{aligned}
& T_{n r m}=\left\{\begin{aligned}
\frac{p_{n r m}}{1-p_{n, m}\left|r_{r n o d} \overline{p m r m}\right|} & \text { if } n_{n r m} \in G^{\prime} \\
0 & \text { otherwise }
\end{aligned}\right. \\
& T_{\text {int }}=\left\{\begin{aligned}
\frac{p_{i n t}}{\left.1-p_{i n t} \mid \bmod \frac{1}{\text { Fint }}\right)} & \text { if } n_{\text {int }} \epsilon G^{\prime \prime} \\
0 & \text { otherwise }
\end{aligned}\right. \\
& T_{a d v}=\left\{\begin{aligned}
\frac{p_{a d i}}{1-p_{a d v}\left[r \cdot \bmod \frac{1}{p_{a d v}}\right]} & \text { if } n_{\text {adv }} \epsilon G^{\prime \prime \prime} \\
0 & \text { otherwise }
\end{aligned}\right.
\end{aligned}
$$

Where G',G', G',' are set of normal nodes, intermediate nodes , and advances nodes that are not selected as cluster head in last $1 /$ popt rounds.

The main drawback of this protocol is if threshold is not achieved user will not any information from network even all the nodes are dead, protocol doesn't any alert. ,So this type of protocols are not used for that kinds of application where data is continuously required.

\subsection{Etssep (Enhanced Threshold Sensitive Stable}

\section{Election Protocol).}

This protocol was proposed by Shekhar Kumar, Shashi Kant Verma And Awadhesh Kumar[12].It is based on dynamically changing cluster head probabilities. The selection of cluster head are on the basis of residual energy level of node and minimum number of cluster heads per round. It is based on TSEP. It is cluster based reactive routing protocol with three-level of heterogeneity i.e normal nodes, intermediate node and advance node. The calculated probabilities depends on the residual energy of node and average energy of network at round $r$. the average energy at round $\mathrm{r}$ is given as 
$\mathrm{E}(\mathrm{r})=$ Etotal $(1-\mathrm{r} / \mathrm{R}) \mathrm{N}$, where $\mathrm{r}=$ current round

$\mathrm{R}=$ total round of network,

$\mathrm{N}=$ total number of nodes and

Etotal= initial energy of network.

The calculated probabilities of nodes are as follows.

$$
\text { Pnrm }=\frac{\text { popt } \cdot E(r i)}{(1+m \cdot \alpha+b \mu) \cdot E(r)}
$$

$$
\text { Pint }=\frac{p o p t \cdot(1+\mu) E(r i)}{(1+m \cdot \alpha+b \mu) \cdot E(r)}
$$

$\operatorname{Padv}=\frac{\text { popt } \cdot(1+\alpha) E(r i)}{(1+m \cdot \alpha+b \mu) \cdot E(r)}$

Where $\mathrm{E}(\mathrm{ri})=$ Residual energy of node.

Simulation using matlab shows that ETSSEP yields $33.5 \%$ more stable than TSEP and double than SEP.

\subsection{Edeec(Enhanced Distributed Energy Efficient}

\section{Clustering)}

EDEEC was proposed by Parul Saini and Ajay K. Sharma[13] which works on same principal of DEEC but is 3-level heterogeneity i.e normal nodes, advance nodes and super nodes. Super nodes have $(1+b)$ times more energy than normal node while advance nodes have( $1+a)$ times more as compared to normal nodes energy. Since it follows same principal of threshold calculation so election probabilities of normal and advance nodes are same, only there is addition of election probabilities of super nodes

$$
p=\left\{\begin{array}{l}
\frac{p_{o p t} E_{i}(r)}{1+m\left(a+m_{0} b\right) E(r)} \text { for normal nodes; } \\
\frac{p_{o p t}(1+a) E_{i}(r)}{1+m\left(a+m_{0} b\right) E(r)} \text { for advanced nodes; } \\
\frac{p_{o p t}(1+b) E_{i}(r)}{1+m\left(a+m_{0} b\right) E(r)} \text { for Super nodes; }
\end{array}\right.
$$

The simulation result shows that this protocol is better than previous protocol and also increase level of heterogeneity.

\subsection{EDDEEC (Enhanced Developed Distributed}

\section{Energy Efficient Clustering)}

EDDEEC protocol was proposed by N.Javaid, T.N Qureshi, A.H khan , A.Iqbal,E.Akhtar And M.Ishfaq[14] which also work on three -level heterogeneity and it overcomes the drawbacks of EDEEC.IN EDEEC ,since super node have highest energy level the election probabilities equation shows that chance of electing as cluster head are more probable in super node as compared to advance node and normal node. So electing as cluster head again-again the energy level of super nodes after some rounds get degraded and have energy same energy level as advance nodes as cluster head consume more energy than other in network .Since super node have same energy level as advanced node but due to high probabilities than advance node ,the super node is again elected as cluster head. So this result in depleting of energy in super nodes and soon they result in dead thereby decreasing network lifetime. This is known as penalizing Effect in EDEEC. So to overcome this effect we consider one parameter known as absolute threshold(Tabsolute). All the nodes use their own election probability until it reaches Tabsolute. When energy level of nodes reaches Tabsolute or below, then we use common election probability for election process.

$$
\text { If } \begin{aligned}
E_{i}(r) & \leq T_{\text {absolute, then }} \\
\qquad p & =c \frac{p_{o p t}(1+b) E_{i}(r)}{1+m\left(a+m_{0} b\right) E(r)} \text { for all nodes }
\end{aligned}
$$

\section{And $\mathrm{T}_{\text {absolute }}=\mathrm{zE}_{\mathrm{o}}$}

Where $\mathrm{z} €[0,1]$. When $\mathrm{z}=0$ it behave as traditional EDEEC. So estimation of closest value of $\mathrm{Z}$ is preferable for increasing first dead node in network. The value of $c$ controls the number of cluster head in the network.when $\mathrm{c}$ value is very high or zero it behaves like direct communication. So appropriate value of $\mathrm{c}$ is calculated for better network performance .Simulation shows that EDDEEC have more effective in respect of stability and network life than earlier protocol.

\subsection{Beenish (Balanced Energy Efficient Network}

\section{Integrated Super Heterogeneous Protocol)}

BEENISH protocol was anticipated by N.Javaid, T.N Qureshi, A.H khan, A.Iqbal,E.Akhtar And M.Ishfaq[15] by increasing heterogeneity-level from their previous protocol i.e four -level of heterogeneity i.e normal node ,advance node , super node, and ultra -super node . The ultra - super node have $(1+u)$ times more energy than normal node. Due to increase in heterogeneity level there is increase in stability period of network .BEENISH uses same election probability as used in previous protocol, beside one extra for ultra-super node the election probabilities of nodes are stated by following formula:-

$\mathrm{p}(\mathrm{i})=$

$$
\begin{aligned}
& \int \frac{\text { popt } \cdot E i}{(1+m(a+m 0(-a+b+m 1(-b+u))) \cdot E a} \text { for norm nodes if } E i>T a b \\
& \frac{p o p t \cdot E \bar{E} \cdot(1+a)}{1+m(a+m 0(-a+b+m 1(-b+u))) \cdot \bar{E} a} \text { for adv nodes if } E(i)>T a b \\
& \left\{\begin{array}{l}
\frac{P O p t+E i *(1+b)}{1+m(a+m 0(-a+b+m 1(-b+w))) \cdot E a} \text { for sup nodes if } E(i)>T a b \\
\frac{p o p t \cdot E i \cdot(1+u)}{1+m(a+m 0(-a+b+m 1(-b+u))) \cdot \bar{E} a} \text { for ult nodes if } E(i)>T a b \\
\frac{c \cdot P o p t \cdot E i \cdot(1+u)}{1+m(a+m 0(-a+b+m 1(-b+u))) \cdot \bar{E} a} \text { for all nodes if } E(i) \leq T a b
\end{array}\right.
\end{aligned}
$$

Tab $=$ Absolute threshold.

Simulation proved that BEENISH is most efficient protocols when compared to DEEC,DDEEC,EDEEC in respect of stability, network lifetime and throughput. 
Comparison Of Different Routing Protocols

\begin{tabular}{|l|l|l|l|l|}
\hline protocols & $\begin{array}{l}\text { Hetero } \\
\text { geneity } \\
\text { level }\end{array}$ & $\begin{array}{l}\text { Stabilit } \\
\text { y } \\
\text { period } \\
\text { (Numb } \\
\text { er of } \\
\text { Round } \\
\text { ) }\end{array}$ & $\begin{array}{l}\text { Networ } \\
\text { k } \\
\text { Lifetim } \\
\text { e } \\
\text { (Numbe } \\
\text { r of } \\
\text { Rounds) }\end{array}$ & Drawback \\
\hline SEP & 2 & 935 & LOW & $\begin{array}{l}\text { Global } \\
\text { knowledge } \\
\text { of nodes is } \\
\text { required }\end{array}$ \\
\hline DEEC & 2 & 1103 & LOW & $\begin{array}{l}\text { Penalizing } \\
\text { Effect }\end{array}$ \\
\hline DDEEC & 2 & 1367 & $\begin{array}{l}\text { MODE } \\
\text { RATE }\end{array}$ & $\begin{array}{l}\text { Valid for 2- } \\
\text { level only }\end{array}$ \\
\hline TSEP & 3 & 1262 & $\begin{array}{l}\text { MODE } \\
\text { RATE }\end{array}$ & $\begin{array}{l}\text { Event } \\
\text { triggered } \\
\text { only }\end{array}$ \\
\hline ETSSEP & 3 & 1402 & HIGH & $\begin{array}{l}\text { Penalizing } \\
\text { Effect }\end{array}$ \\
\hline EDEEC & 3 & 1421 & HIGH & $\begin{array}{l}\text { Penalizing } \\
\text { Effect }\end{array}$ \\
\hline EDDEEC & 3 & 1717 & HIGH & $\begin{array}{l}\text { Valid for 3- } \\
\text { level only }\end{array}$ \\
\hline BEENISH & 4 & 1661 & HIGH & $\begin{array}{l}\text { Penalizing } \\
\text { Effect }\end{array}$ \\
\hline
\end{tabular}

\section{MOBILITY BASED ROUTING POTOCOL}

Introduction of mobility parameter in WSNs make more challenging issue while designing routing protocols for wireless sensor networks[16]. Due to introduction of mobility of sink node all our protocols we discussed so far get out of the picture as we consider static sink node while designing the above protocols. Mobility of sink nodes requires energy -efficient protocols as well gaureenting of data delivery from source sensor node to mobile sink node. While designing mobility based protocol designer must keep these parameter in mind such as error, noise ,interference, random topology, guaranteed of data delivery and shortest route etc. .Some mobility based routing protocols are as follow:-

\subsection{Joint Mobility and Routing Protocol:}

A WSNs with static sink node suffer from a commonly problem known as energy sink -hole problem. This problem state that all the sensor nodes which are located around static sink nodes are continuously used for forwarding data to the sink node on behalf of other nodes. Thus it result in high depletion of source sensor node energy that are close to sink node due to heavy load and soon result in death of sensor node .To compensate this problem, we use concept of mobile sink node is used instead of static sink node[17] .In this concept the sensor node surrounding the sink node changes over the time by giving to all sensor node to act as relay node and thus maintaining load balancing of data routing among the sensor nodes. Here we uses concept of shortest path routing mechanism for effective and timelimited data delivery. The movement of sink node also play major role in routing of data .If we considered area of deployment as circular field then trajectory movement of sink node yields better performance. We can also use centric and annuli movement but trajectory is preferable.

\subsection{Data MULES Based Protocol:-}

This protocol was proposed to concentrate on the need of reliable and cost effective connectivity in sparse network by reducing energy consumption[18]. It is based on mobile device called Mobile ubiquitous LAN extensions(MULE).The architecture of MULE contain 3 layer.yhe lower layer include static wireless sensor nodes whose duty is to sense the environment. The top layer contain WAN connected units and access point that is used for analyzing the sensed data. These access points communicates with central data warehouse and enabling them for synchronization of collected data, identification of redundant data and acknowledgment of data send by MULEs for reliable data transmission. Middle layer has mobile units(MULEs) that move across sensor field and gather data from sensor nodes and deliver to access point which are in close range. MULE architecture help in saving energy as there is direct communication between MULEs and sensor node through shortest path, so energy degraded takes place slowly and uniformly result in increasing network lifetime. Also there is no routing overhead . but failure of MULEs unit result increasing latency and decreasing data success rate in degradation of performance of sparse network. For time critical application we use always on connection mode for better response.

\subsection{Scalable Energy -Efficient Asynchronous Dissemination(SEAD):-}

It was Self-organizing protocol which focus on minimization of delay to sink node and savings of energy level[19]. SEAD uses data dissemination concept in which sensor nodes reports its sensed data to multiple sink node which come under its range. It usually take place in 3 step i.e dissemination tree construction(d-tree), data dissemination and maintenance of links to sink nodes. The protocol consider a assumption that all sensor nodes have knowledge of their geographic location. Every sensor node creates it d-tree rooted at itself and for all nodes it is built separately. It is also categorized as location aware routing protocols.

\subsection{Dynamic Proxy Tree-Based Data}

\section{Dissemination:-}

This protocol was proposed for creating a dynamic proxy tree in which there is tree connecting a source sensor to all its interested multiple sink node[20]. This will help source node to circulate its data directly to mobile sink nodes. In 
protocol we consider stationary source node while sink nodes are mobile in nature. Sensor node are continuously used to detect and monitor some mobile targets, where mobile sink nodes are used to gather data from source nodes whose duty is to aggregates data from set of sensor node. Due to mobility of sink node source node changes from time to time as new sensor closer to target mobile node become source node. Both source sensor and mobile sink node have proxies known as stationary source proxy and stationary sink proxy. These proxies are stationary in nature because source nodes changes while sink nodes moves. When the distance between source and its current proxies exceeds an amount of threshold, it is assign a new proxies. Similarly when distance between sink node and its current proxies exceeds threshold. This protocol help in curtailing cost of querying and pushing of data from source node to sink node.

\section{CONCLUSION AND FUTURE SCOPE}

Heterogeneity and Mobility issues are major factor that must be fulfilled by designer while designing routing protocols for WSNs. In this survey paper, we tried our best to present both heterogeneous and mobility based routing protocols. We also tried to make comparative study of heterogeneous routing protocol based on performance metric like stability period, network lifetime, level of heterogeneity etc. .We also present concept of mobile sink node and multiple sink nodes for better understanding of mobility issues.

For future work we can consider multi-level heterogeneity for improving stability and network life-time of network. We can also consider the mobility movement of sink nodes with respect to cluster head node for efficient energy routing protocols. We can also consider multi-level heterogeneity with multi-mobile sink nodes.

\section{REFERENCES}

[1]. "21 ideas for the $21^{\text {st }}$ century", Business Week, Aug. 301999 , pp. 78-167.

[2]. Jun Zheng and Abbas Jamalipour, "Wireless Sensor Networks: A Networking Perspective", a book published by A John \& Sons, Inc, and IEEEE, 2009.

[3]. S.K. Singh, M.P. Singh, and D.K. Singh, "A survey of Energy-Efficient Hierarchical Cluster-based Routing in Wireless Sensor Networks", International Journal of Advanced Networking and Application (IJANA), Sept.-Oct. 2010, vol. 02, issue 02, pp. 570-580..

[4]. W. R. Heinzelman, "Application-Specific Protocol Architectures for Wireless Networks," Ph.D. thesis, Massachusetts Institute of Technology, 2000.

[5]. E. J. Duarte-Melo and M. Liu, "Analysis of energy consumption and lifetime of heterogeneous wireless sensor networks," in Proceedings of Global Telecommunications Conference (GLOBECOM 2002). IEEE, November 2002, pp. 21-25.

[6]. V. Mhatre and C. Rosenberg, "Homogeneous vs. heterogeneous clustered sensor networks: A comparative study," in Proceedings of 2004 IEEE
International Conference on Communications (ICC 2004), June 2004.

[7]. H. O. Tan and I. Korpeoglu, "Power efficient data gathering and aggregation in wireless sensor networks," SIGMOD Record, vol. 32, no. 4, pp. 6671, 2003.

[8]. G. Samaragdakis, I. Matta, A. Bestavros, "SEP: A Stable Election Protocol for clustered heterogeneous wireless sensor network", in: Second International Workshop on Sensor and Actor Network Protocols and Applications (SANPA 2004), 2004.

[9]. L. Qing, Q. Zhu, M.Wang, "Design of a distributed energy-efficient clustering (DEEC)algorithm for heterogeneous wireless sensor network", ELSEVIER, Computer Communications 29, 2006, pp2230-2237.

[10]. B. Elbhiri, Saadane Rachid, S El Fkihi, D Aboutajdine, "Developed Distributed EnergyEfficient Clustering (DDEEC) for heterogeneous wireless sensor networks", in: 5th International Symposium on $\mathrm{I} / \mathrm{V}$ Communications and Mobile Network (ISVC), 2010.

[11]. A,Kashaf., N. Javaid. A.Z khan , \& I.A.Khan. (2012). "TSEP: Threshold-sensitive stable election protocol for WSNs. In 10th international conference on frontiers of information technology (FIT) (Vol. 164, no. 168, pp. 17-19)."

[12]. Shekhar Kumar ,Shashi Kant Verma and Awadhesh Kumar "ETSSEP: Enhanced Threshold Sensitive Stable Election Protocol for Heterogeneous Wireless Sensor Network “ Springer ,2013.

[13]. Parul Saini, Ajay.K.Sharma, "E-DEEC- Enhanced Distributed Energy Efficient Clustering Scheme for heterogeneous WSN", in: 2010 1st International Conference on Parallel, Distributed and Grid Computing (PDGC - 2010).

[14]. N. Javaid, T. N. Qureshi, A.H. Khan, A. Iqbal, E. Akhtar and M. Ishfaq, "EDDEEC: Enhanced Developed Distributed Energy-Efficient Clustering for Heterogeneous Wireless Sensor Networks", ELSEVIER, Procedia Computer Science 00, 2013, pp 1-6.

[15]. N. Javaid, T. N. Qureshi, A.H. Khan, A. Iqbal, E. Akhtar and M. Ishfaq, "BEENISH: Balanced Energy Efficient Network Integrated Super Heterogeneous Protocol for Wireless Sensor Networks", ELSEVIER, Procedia Computer Science 19, 2013, pp 920-925.

[16]. Shio Kumar Singh , M P Singhand D K Singh

[17]. "A Survey -Routing Protocols in Wireless Sensor Networks "Nov- 2010.

[18]. J. Luo, and J.- P. Hubaux, "Joint mobility and routing for lifetime elongation in wireless sensor networks", Proceedings IEEE INFOCOM'05, vol. 3, Miami, FL, Mar. 2005, pp. 1735-1746.

[19]. R.C. Shah, S. Roy, S. Jain, and W. Brunette, "Data MULEs: Modeling a three-tier architecture for sparse sensor networks ", Proceedings SN P A '03, Anchorage, AK, May 2003, pp. 30-41. 
[20]. B. Karp and H. T. Kung, "GPSR: Greedy perimeter stateless routing for wireless networks",Proceedings ACM MobiCom'00, Boston, MA, Aug. 2000, pp. 243-254.

[21]. W. Chang, G. Cao, and T. La Porta, "Dynamic proxy tree-based data dissemination schemes for wireless sensor networks", Proceedings IEEE MASS'04, Fort Lauderdale, FL, Oct. 2004, pp. 21-30. 